\title{
Development of a Gas-Solid Drag Law for Clustered Particles Using Particle-Resolved Direct Numerical Simulation
}

\author{
Mohammad Mehrabadi, Eric Murphy, Shankar Subramaniam* \\ Center for Multiphase Flow Research, Department of Mechanical Engineering, Iowa \\ State University, Ames, IA 50011, USA
}

\begin{abstract}
Particle-resolved direct numerical simulation (PR-DNS) is used to quantify the drag force on clustered particle configurations over the solid phase volume fraction range of $0.1 \leq \phi \leq 0.35$ and the mean slip Reynolds number range of $0.01 \leq R e_{m} \leq 50$. The particle configurations and flow parameters correspond to gas-solid suspensions of Geldart A particles in which formation of clusters have been reported. In our PR-DNS, we use clustered particle configurations that match cluster statistics observed in experimental studies. To generate the particle configurations, we perform discrete element method (DEM) simulations of homogeneous cooling gas (HCG) systems with cohesive and inelastic particles in the absence interstitial fluid. Clustered particle subensembles are then extracted from HCG simulations to match the statistics of cluster size distributions observed in experiments. These sub-ensembles are used for PR-DNS. It is found that the mean drag on clustered configurations decreases when compared to the drag laws for uniform particle configura-
\end{abstract}

\footnotetext{
${ }^{*}$ Corresponding author

Email address: shankar@iastate.edu (Shankar Subramaniam)
} 
tions. The maximum drag reduction belongs to the configuration with low solid-phase volume fraction $\phi=0.1$ in Stokes flow, and is about 35\%. The drag reduction reduces with increase in both $\phi$ and $R e_{m}$. A clustering metric is introduced to explain the behavior of the drag reduction with respect to solid-phase volume fraction. Also the behavior of the drag reduction with mean slip Reynolds number is related to the Brinkman screening length. PRDNS results are then used to propose a clustered drag model for the range of flow parameters considered in this study. This clustered drag model provides a smooth transition between the uniform and clustered states by means of a weighting function with two model parameters.

Keywords: Particle-resolved direct numerical simulation, Gas-solid flow, Clustered configuration, Drag law

\section{Introduction}

Gas-solid flows are commonly found in industrial applications such as fluidized-bed combustion, fluid catalytic cracking, coal gasification, and biomass energy generation (Fan et al., 2004). In these applications, there are several mechanisms that tend to bring individual particles close to each other. These particles then form locally denser regions of particles compared to ambient that surrounds them. These structures are known as particle clusters that dynamically form and break up in response to gas-particle and particle-particle interactions in gas-solid flows. The presence of particle clusters significantly influences interphase transfer of mass, momentum and energy in a suspension. For instance, interphase heat and mass transfer in suspensions with particle clusters is significantly reduced when compared to those flows where 


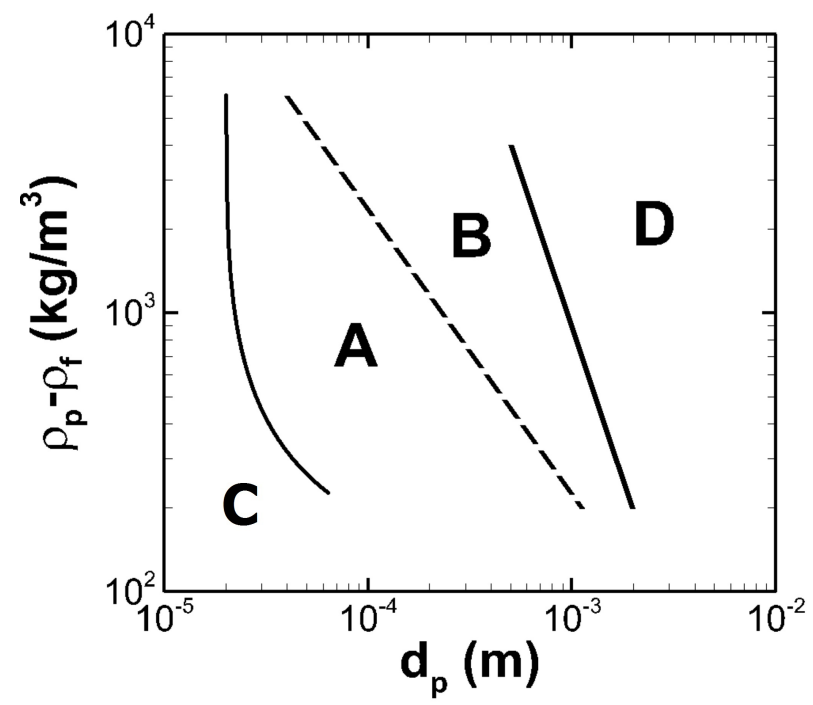

Figure 1: Geldart classification chart based on particle diameter and density difference between the fluid phase and the solid phase.

particles are uniformly distributed in the suspension. Also in fluidized bed risers once particle clusters form the pressure drop along the riser decreases. This indicates a reduction of gas-particle drag when clusters exist in the riser. Therefore, it is essential to account for the presence of these structures when analyzing engineering applications that utilize gas-solid suspensions.

In the gas-solid flow applications mentioned above, the material density ratio ranges from $\rho^{(p)} / \rho^{(f)} \sim \mathcal{O}(100)$ to $\mathcal{O}(1000)$ and the particle diameter varies from $d_{p} \sim 50 \mu \mathrm{m}$ to $500 \mu \mathrm{m}$. In the Geldart classification, gas-solid suspensions are characterized based on particle diameter and the density difference between the two phases. From the Geldart chart shown in Fig. 1 it can be understood that the aforementioned ranges for density ratio and particle diameter belong to Geldart A and Geldart B particles. For Geldart A particles that are associated with smaller and lighter particles, the dominant 


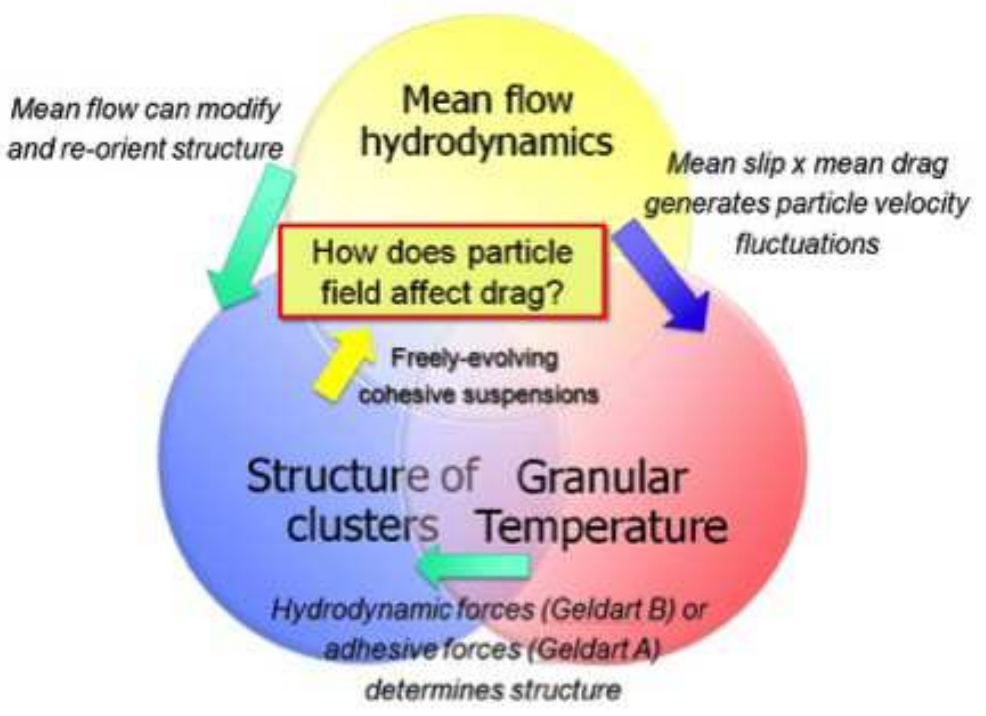

Figure 2: Particle-particle and fluid-particle interactions in a gas-solid flow.

clustering mechanism is particle-particle interaction arising from electrostatics, cohesion, van der Waals forces, or liquid bridging (Gao et al., 2008; van Wachem and Sasic, 2008; Royer et al., 2009), while for Geldart B particles with larger and heavier particles, the effect of hydrodynamic interactions is more dominant.

It is evident from homogeneous cooling gases (HCG) of granular materials that the level of particle velocity fluctuations, also known as particle granular temperature, plays a significant role in the formation of particle clusters. In homogeneous cooling gases as the suspension cools down, locally concentrated regions of particles form in the suspension. In these concentrated regions, the particle collision frequency is higher than in the less concentrated regions. Therefore, the particles in the concentrated regions lose more energy due to higher frequency of inelastic collisions which accelerates the 
cooling of these locally concentrated regions. Since the granular temperature is associated with particle pressure, these highly concentrated cool regions appear as low pressure zones which lead to attraction of more particles and enhancement of particle clustering.

Although the particle granular temperature is an important flow variable that is implicated in clustering of particles, the generation of particle clusters in gas-solid flows arises from a complex dynamic interplay between fluid-particle and particle-particle interactions. In a gas-solid suspension, formation of these particle clusters may arise from the transport of particle relative velocity fluctuations in pair separation space, or from the balance between the attractive forces (hydrodynamics, electrostatics, or cohesion) bringing the particles together and the repulsive forces (hydrodynamics, or collisional forces) trying to break up these structures. The presence of clusters then gives rise to a reduction in the drag force. We have learned from PR-DNS of freely evolving gas-solid suspensions that the rate of work done in maintaining a flow with constant mean slip between the gas and solid phases by means of a constant mean pressure gradient results in the production of velocity fluctuations in both gas and solid phases (Mehrabadi et al., 2015). Therefore, reduction in the local drag force acting on particle clusters leads to local reduction of the amount of power input to the granular temperature. This reduction in granular temperature enhances the formation of clusters. The carrier flow can also directly influence particle clusters by reorienting and restructuring of the clusters. These interconnected phenomena dynamically affect the interphase transfer of momentum and energy between the gas phase and solid particles. The outcome of this complex dynamic interplay is 
growth of particle clusters and formation of mesoscale structures as observed in experiments (Shaffer et al., 2013). Figure 2 schematically describes this interplay in which the mean flow indirectly influences particle clustering by tuning the supply of kinetic energy to the granular temperature. The interplay between dissipation, of hydrodynamic or collisional origin, the granular temperature, and cohesive potentials then determines the formation of clusters. Once the clusters form, they are directly influenced by the carrier flow, in a way that differs from dispersed particles. In addition to this complex mechanism arising from interphase interactions, wall effects, inflow and outflow boundaries, and inhomogeneities in the carrier flow can influence the formation of particle clusters as well, which make the clustering analysis more complicated.

In computational fluid dynamics (CFD) calculations of gas-solid flow, the momentum exchange between the gas phase and the solid phase is modeled by drag closure models. In this regard, empirical correlations based on experiments (Wen and Yu, 1966; Ergun, 1952), or those proposed from particle-resolved direct numerical simulation (Hill et al., 2001a,b; van der Hoef et al., 2005; Beetstra et al., 2007; Tenneti et al., 2011) are widely used to account for the average drag force on particles in gas-solid flows. These drag laws are generally applicable to suspensions with particles uniformly distributed in the suspension. However, the use of these drag laws for cases where particle clusters are likely to form, such as Geldart A suspensions, shows inconsistency between the CFD simulations and experimental data (Zimmermann and Taghipour, 2005; Mazzei and Lettieri, 2008; Garg et al., 2010a). This inconsistency indicates that the closure models proposed for 
uniformly distributed particle configurations are not capable of correctly predicting the unresolved interphase interactions for suspensions where particle clusters form.

The current state-of-the-art drag model that accounts for the effect of particle clusters in CFD calculations is a class of energy minimization multiscale (EMMS) models (Li et al., 1999; Yang et al., 2004; Wang and Li, 2007) that are extensions to conventional drag models, and is based on the minimum required energy for suspending and transporting dense particle regions in a gas-solid suspension. While the EMMS model is useful, there is scope to develop a physics-based model of drag in clusters particle suspensions.

Experimental investigations provide invaluable insight into formation, distribution, interaction and more importantly characterization of particle clusters in gas-solid flows. For instance, Harris et al. (2002) compiled a wide range of experimental and industrial data corresponding to near-wall particle clusters in risers and provided correlations for cluster properties such as cluster volume fractions, cluster diameter, cluster roundness factor, etc. Cocco et al. (2010) used a fiber optical probe to provide cluster size distribution in and above fluidized beds. Also Chew et al. (2012) investigated the formation, duration and frequency of appearance of particle clusters at different operating conditions along a riser for different materials. Gokaltun et al. (2012) used shadow-sizing technique to measure cluster size and velocity distribution in a circulating fluidized bed. Also, McMillan et al. (2013) reported that in a riser although short-range particle-particle forces initiate small clusters, it is the fluctuations and gradients in gas-particle forces that promote cluster formation. 
In spite of the useful information provided by the experiment, detailed spatio-temporal analysis of the flow hydrodynamics and transfer of mass, momentum and energy due to interphase interactions cannot be obtained from experiment. In an experiment it is not easily possible to exclude the wall effects in the flow setup, making it difficult to differentiate the role of wall effects from other mechanisms that contribute to formation of particle clusters.

Particle-resolved direct numerical simulation (PR-DNS) is an alternative approach that is well suited for discovering flow physics in particle-laden flow (Tenneti and Subramaniam, 2014). The PR-DNS approach not only provides detailed information about the hydrodynamic velocity and pressure fields, it also provides particles trajectories along with their velocities and accelerations. Furthermore, properly designed PR-DNS studies can be used to either propose closure models used in CFD calculations of gas-solid flow, or to test the validity of these closure models.

The PR-DNS methodology has been successfully used to propose closure models for the average gas-particle drag. Hill et al. (2001a) used the Lattice Boltzmann Method (LBM) to propose a drag model for monodisperse arrays of ordered and randomly distributed particles in the Stokes flow regime, and validated their results for low solid volume fraction with theoretical analysis. Later, Hill et al. (2001b) extended their drag model to moderate Reynolds numbers. van der Hoef et al. (2005) also used LBM to a propose drag model for monodisperse gas-solid flow in the Stokes regime using fixed particle assemblies. Beetstra et al. (2007) used LBM to solve for flow past fixed particle assemblies to incorporate the effect of higher mean slip Reynolds number (up 
to 1000) into the low-Reynolds-number monodisperse drag model of van der Hoef et al. (2005). Similarly, Rong et al. (2013) used LBM simulations to propose a drag in the range of the solid-phase volume fraction $0.1 \leq \phi \leq 0.6$ and mean slip Reynolds number $0.002 \leq R e_{m} \leq 3000$. Tenneti et al. (2011) performed well-resolved PR-DNS based on the particle-resolved Reconcilable Immersed Boundary Method (PUReIBM) continuum solver to simulate the Navier-Stokes equations and reported the drag force in gas-solid flows using fixed particle assemblies over a wide range of flow parameters $(0.1 \leq \phi \leq 0.5$ and $\left.0.01 \leq R e_{m} \leq 300\right)$. They also proposed a drag law as a function of $\phi$ and $R e_{m}$.

In an attempt to generalize the quantification of interphase momentum transfer to clustered particle configurations using PR-DNS, Beetstra et al. (2006) performed simulations for four types of particle clusters which were originally constructed by Tran-Cong et al. (2004) for experimental analysis. Based on their PR-DNS results, Beetstra et al. (2006) reported that the interstitial distance among particles in a cluster has significant effect on the drag force, and neglecting this parameter leads to inaccurate drag prediction. Shah et al. (2013) also used LBM to study the effect of clusters on the drag force. They successfully demonstrated the dependence of the drag on flow Reynolds number and solid-phase volume fraction. However, in their configurations they used a stand-alone cluster in the middle of the computational box surrounded by individual particles. This type of particle configuration may not accurately represent the configurations observed in real applications, because the clustered configurations obtained from this deterministic approach may not represent the statistics of clusters observed in experiment. 
In spite of the initial insight about the effect of clustering on the drag force, the aforementioned PR-DNS works neglected the simultaneous coexistence of different cluster types that lead to a cluster size distribution in a gas-solid flow. Experimental analysis of Cocco et al. (2010) in fluidized bed shows that a significant percentage of particles participate in formation of clusters ranging from a few to hundreds of particles in each cluster. The shape of the distribution may depend on particle materials, operating conditions and flow location. Also particle clusters appearing as a result of hydrodynamic interactions may have geometrical characteristics that have not yet been extensively addressed. Therefore, those analysis that do not account for clustering metrics may have limited applicability.

In the current study we first describe different types of PR-DNS for clustered configurations, and analyze their numerical feasibility by performing a timescale analysis. Then, in order to have clustered configurations that better match real applications, we perform homogeneous cooling gas simulation of inelastic and cohesive particles using discrete element method (DEM) and extract particle configurations that are statistically equivalent to the distribution provided by the experiment of Cocco et al. (2010). In order to identify the level of clustering at different solid-phase volume fractions, we propose a clustering metric that expresses the deviation of particle configuration from a uniformly distributed state. These clustered configurations are then used for PR-DNS of gas-solid flow over a rage of flow parameters and the results are used to provide a drag model for clustered configurations. 


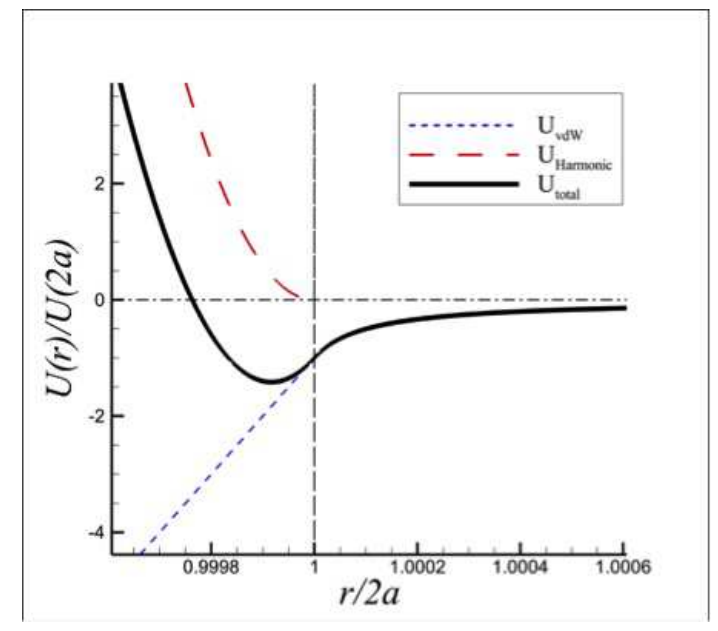

Figure 3: Depiction of the functional form of particle-particle interaction potentials. The Harmonic potential representing the particle-particle interaction due to elastic collision is shown in long dashed lines. The van der Waals potential representing the cohesive force is shown in short dashed lines. The total interaction potential obtained from superposition of the Harmonic and van der waals potentials in shown in solid line.

\section{Analysis of numerical constraints}

In PR-DNS of freely evolving suspensions, particle collisions are treated with the soft-sphere model that allows finite overlap of particles during the collision process. This model typically employs a linear spring-dashpot to represent the contact force that results in elastic or inelastic restitution on contact (Cundall and Strack, 1978), though more physics based contact force model alternatives are also used (Kuwabara and Kono, 1987). The attractive potential that we consider between a pair of particles not yet in contact is modeled by the van der Waals (VDW) potential given by

$$
U_{\mathrm{coh}}(r)=-\frac{A d_{p}}{24\left(r+d_{0}-d_{p}\right)},
$$


where $A$ is the Hamaker constant, $d_{p}$ is the particle diameter, $d_{0}$ is the interatomic distance, and $r$ is the particle center to center distance. The attractive force saturates once particles are in contact. Figure 3 shows the spring $U_{\text {Harmonic }}$ and cohesion potentials $U_{\text {VDW }}$ for a pair of cohesive particles undergoing an elastic collision, and the resulting total potential $U_{\text {total }}$. Note that the cohesive force at contact, $r<0$, is constant. In order to resolve the motion of a pair of particles going through this pair potential in a discrete element method (DEM) simulation, two timescales should be resolved. The first one is the collision timescale defined as

$$
t_{\mathrm{coll}}=\pi \sqrt{\frac{m}{k}}
$$

where $m$ is the effective mass of the particle pair and $k$ is the spring stiffness. We can derive an expression for this timescale normalized by the characteristic timescale of the fluid $t_{\text {fluid }}=d_{p} /|\mathbf{W}|$ by writing the energy balance between the spring potential energy and the particle kinetic energy

$$
\frac{1}{2} k \delta^{2}=\frac{1}{2} m v^{2}
$$

where $\delta$ is the overlap distance, and $v$ is the characteristic velocity of colliding particles. In a gas-solid flow this characteristic velocity can be considered as the second root of solid-phase granular temperature $v=\sqrt{T}$, where $T=$ $\left\langle\mathbf{v}^{\prime \prime} \cdot \mathbf{v}^{\prime \prime}\right\rangle / 3$ with $\mathbf{v}^{\prime \prime}$ being particles velocity fluctuations, i.e., $\mathbf{v}^{\prime \prime}=\mathbf{v}-\langle\mathbf{v}\rangle$. Now if the velocity in Eq. 3 is replaced with $\sqrt{T}$ and both sides of the equations are multiplied by $\left(|\langle\mathbf{W}\rangle| / d_{p}\right)^{2}$, Eq. 3 becomes

$$
\frac{m}{k}\left(\frac{|\langle\mathbf{W}\rangle|}{d_{p}}\right)^{2}=\frac{|\langle\mathbf{W}\rangle|^{2}}{T}\left(\frac{\delta}{d_{p}}\right)^{2} .
$$


If one divides the left-hand side by the right-hand side, it is evident the ratio of contact to fluid timescale scales with the non-dimensional overlap $t_{\text {coll }} / t_{\text {fluid }} \propto \delta / d_{p}$. The model presented herein assumes nearly rigid particles $\delta / d_{0}<O(1)$ (Derjaguin et al., 1975) and hence very small overlaps. Additionally, it has been found that physically accurate simulations require large spring stiffness, on the order of the stiffness of actual particles (Murphy and Subramaniam, 2016a). As a result very little speed-up can be achieved through softening of the interaction without affecting the physics.

While the limiting timescale for these simulations is the collision timestep, we can still gain an appreciation of the cost of such numerical simulations by looking at the cohesive timescale. The cohesive timescale is the timescale that must be resolved in order to enforce energy conservation between particles as they move through the attractive potential well as shown in Fig. 3. Now if we assume that in Eq. 4 the maximum overlap $\delta$ is $1 \%$ of the particle diameter $d_{p}$ and also assume that the granular temperature in gas-solid flow scales as $T /|\langle\mathbf{W}\rangle|^{2} \sim 10^{-2}$ (Tenneti et al., 2016), it can be concluded from Eq. 4 that the contact to fluid timescales ratio is

$$
\frac{t_{\text {coll }}}{t_{\text {fluid }}} \sim 0.1 .
$$

The second important timescale in systems with attractive forces, particularly with cohesive forces in the current analysis, is the cohesion timescale that should also be resolved to capture the motion of a colliding pair through the particle-particle well potential as shown in Fig. 3. The cohesion timescale relative to the fluid timescale is defined as (Murphy and Subramaniam, 
2016a)

$$
\frac{t_{\text {coh }}}{t_{\text {fluid }}}=\frac{d_{0}}{d_{p}} \frac{|\langle\mathbf{W}\rangle|}{T^{1 / 2}} \sqrt{\frac{2000}{H a+40}},
$$

where the non-dimensional $\mathrm{Ha}$ parameter describes the ratio of the energy associated with the cohesive forces to the characteristic kinetic energy in a pair of colliding particles, which is given as

$$
H a=\frac{A}{2 \rho_{p} \pi d_{p}^{2} d_{0} T},
$$

where $\rho_{p}$ is the particle mass density. For a given cohesive and colliding system with $A=10^{-19} \mathrm{~J}, \rho_{p}=400 \mathrm{~kg} / \mathrm{m}^{3}, d_{0}=2$ Angstroms, $d_{p}=50$ microns, $T=0.00085 \mathrm{~m}^{2} / \mathrm{s}^{2}$, the $H a$ parameter appears to be about 0.1 . For this system, the cohesion to fluid timescales ratio in Eq. 6 is

$$
\frac{t_{\text {coh }}}{t_{\text {fluid }}} \sim 0.003
$$

The above timescale ratio indicates that the cohesion timescale is about two orders of magnitude smaller than the collision timescale for the softened systems normally simulated in gas-solid flows, $\delta / d_{p}$, as calculated by Eq. 5 . From this analysis the simulation time for PR-DNS of gas-solid systems with cohesive particles is at least two orders of magnitude longer than a similar system in the absence of cohesive forces.

Based on the preceding timescale analysis provided, we identify three simulation approaches for PR-DNS of flow past particle clusters:

1. PR-DNS of homogeneous freely evolving gas-solid suspensions of cohesive particles from an initially unclustered state where the cohesion timescale given by Eq. 8 is resolved 
2. PR-DNS of homogeneous freely evolving suspensions of initially clustered particles that still resolves the cohesion timescale but eliminates cluster formation time

3. PR-DNS of homogeneous fixed assemblies of particle clusters where the cohesion and collision timescales are irrelevant because the particles do not move

The first approach that involves the formation of clusters from a uniform initial state accounting for both cohesive and hydrodynamic forces is computationally expensive owing to the range of time and length scales needed to be resolved. Note that we have found from DEM of HCG that the formation time is long (hundreds of millions of time steps is required). Since the cohesive to contact and cohesive to hydrodynamic force ratios are important in Type 1 simulations, a scaling analysis is provided in Appendix A accordingly. The second approach is useful for studying the restructuring and reorientation of clusters in a gas-solid flow, but although it avoids the simulation of the time to form clusters, it is still expensive for parametric studies using PR-DNS. The third approach is the most inexpensive and expedient one to investigate the effect of particle clusters on flow hydrodynamics. The use of rigid clusters or fixed particle assemblies implies that the cohesive forces among particles in a cluster overcome the hydrodynamic forces experienced by particles that tend to break clusters apart; the particles are effectively 'sintered' into clusters. Therefore, the focus of this study is on the third approach. It has been shown that fixed particle assemblies of high Stokes number particles in PR-DNS are a good approximation to freely evolving suspensions $(\mathrm{Xu}$ and Subramaniam, 2010; Tenneti et al., 2011; Mehrabadi et al., 2015), be- 
cause the time required for the particle configuration to change significantly is much larger than the fluid relaxation timescale.

Therefore, in this work we use the third approach to study the effect of particle clustering on gas-particle drag over a range of mean slip Reynolds number and solid-phase volume fraction. These clusters are associated with Plascoat polyethylene particles based on previously established analysis (Cocco et al., 2010) that predicts the maximum size of clusters under the influence of interparticle attractions and flow hydrodynamics. The computational generation of particle clusters with statistics that match experimental findings is discussed in the following section.

\section{Initialization of clustered particle configuration}

Characterization of particle clusters and relating this characterization to flow parameters as well as particle properties is a challenging task. The dynamic formation and breakup of particle clusters usually leads to the existence of a range of particle cluster sizes in the system. Although some correlations for the size, concentration and shape of particle clusters have been proposed for some specific flow conditions such as core-annular flow

(Harris et al., 2002), the dynamics of clusters as well as their corresponding size and shape distributions in engineering applications is an open question. In this regard, the experimental work of Cocco et al. (2010) provides detailed information about the cluster size distribution of Plascoat polyethylene and FCC catalyst particles in a fluidized bed. They used a specially designed borescope connected to a high speed camera that was inserted into the fluidized bed. Images from the fluidized bed were post-processed to provide 
information about particles and clusters. Their analysis for polyethylene particles indicates that there exists a cluster size distribution in the bed. Although there are clusters with hundreds of particles, the mean number of particles in a cluster is about 26 . In addition, about $75 \%$ of particles form clusters, while the rest appear isolated particles. Their measurement for FCC particles showed similar findings. However, the mean number of particles in a FCC cluster was reported as 13. The experimental findings of Cocco et al. (2010) indicate that those studies in which the interaction of the carrier flow with a single particle cluster (Beetstra et al., 2006) or a single particle cluster with surrounding individual particles (Shah et al., 2013) may have limited applicability in interphase interactions in a practical situation where there is a cluster size distribution in the system.

In this study we use PR-DNS of clustered configurations that match the statistics of particle clusters represented in experimental findings. PR-DNS of gas-solid flow for the entire range of cluster size distribution provided by Cocco et al. (2010) requires huge computational resources. Instead, in the current study we quantify the drag force on sub-ensembles of particle clusters that have been extracted from DEM simulation of a HCG with both inelastic and cohesive particles in the absence of interstitial fluid. We select the extracted sub-ensembles to be consistent with the results of Cocco et al. (2010) for polyethylene particles as the baseline for the particle configurations in our PR-DNS. We expect these sub-ensembles to meet the following constraints:

1. The particle volume fraction in the sub-ensembles should be close to that of the HCG system

2. Particle structures in the sub-ensembles have the same statistics as 
those in the whole HCG system

3. The maximum number of particles in the largest cluster of the subensemble be around 26

4. Approximately $75 \%$ of particles in the sub-ensemble are in clusters

HCG simulations are performed using the LAMMPS code (Plimpton, 1995) developed in Sandia National Lab. These simulations are performed in a periodic computational box in the same way as documented in (Murphy and Subramaniam, 2015). The simulations are performed in a domain size with $\mathcal{L} / d_{p}=50$ for solid-phase volume fractions in the range $0.1 \leq \phi \leq 0.4$. The number of particles can be determined by the following expression:

$$
N_{p}=\frac{6 \phi}{\pi}\left(\frac{\mathcal{L}}{d_{p}}\right)^{3} .
$$

Particles are initially uniformly distributed in the domain with a given Maxwellain velocity distribution that is characterized by granular temperature $T$. The short-range interaction forces among particles are represented by the van der Waals force arising from the well potential given by Eq. 1. Also the particle collisions in these HCG simulations are modeled by a soft-sphere method using the nonlinear spring-dashpot model of Kuwabara and Kono (1987). In the absence of any external forces, particles lose energy as they undergo inelastic particle collisions. Once the kinetic energy of a pair of colliding particles is not enough to overcome the energy in the well potential described by Eq. 1, these particles become bound, eventually sticking together and forming clusters. This process progressively leads to formation of larger particle clusters in the domain since there is no mechanism to compensate the energy loss in inelastic particle collisions, and therefore, eventually all particles fall into a giant cluster that spans through the computational box. 


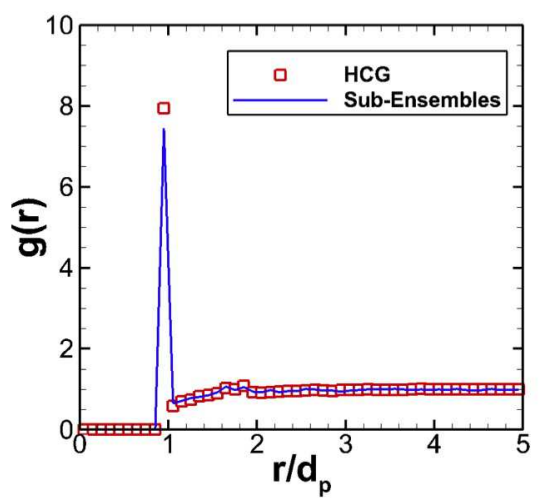

(a)

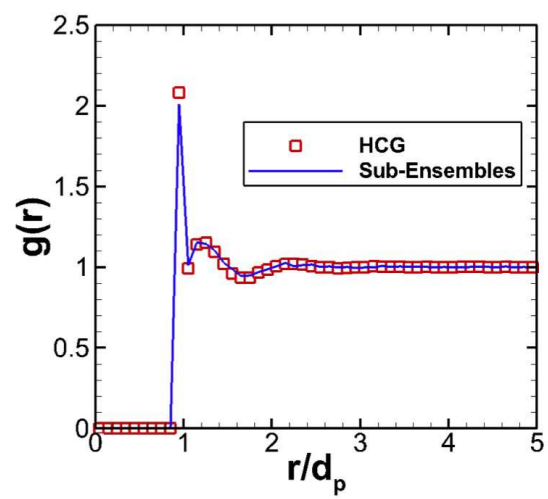

(b)

Figure 4: Radial distribution function of the HCG system compared to that obtained from the sub-ensembles for (a) $\phi=0.1$, and (b) $\phi=0.3$.

At various time instants during the HCG simulation, the systems are searched during the cooling process for particle sub-ensembles that meet the constraints mentioned above and have the box length as $\mathcal{L} / d_{p}=10$. Due to the periodic image of particle configurations in sub-ensembles, there may be overlap of particles near the boundaries. For those particle pairs that overlap more than $5 \%$ of a particle diameter, one of the particles in the pair is removed. It should be noted that this process leads to sub-ensembles that have slightly smaller volume fractions (1 to 10\%) compared to the original HCG systems. Figure 4 shows the comparison between the radial distribution function $g(r)$ of the HCG system with the averaged $g(r)$ obtained from the sub-ensembles for the cases with solid-phase volume fractions $\phi=0.1$ and 0.3. The radial distribution function represents the probability of finding a particle in the neighborhood of a test particle with separation $r$ between particles centers compared to that of a completely random Poisson process. 

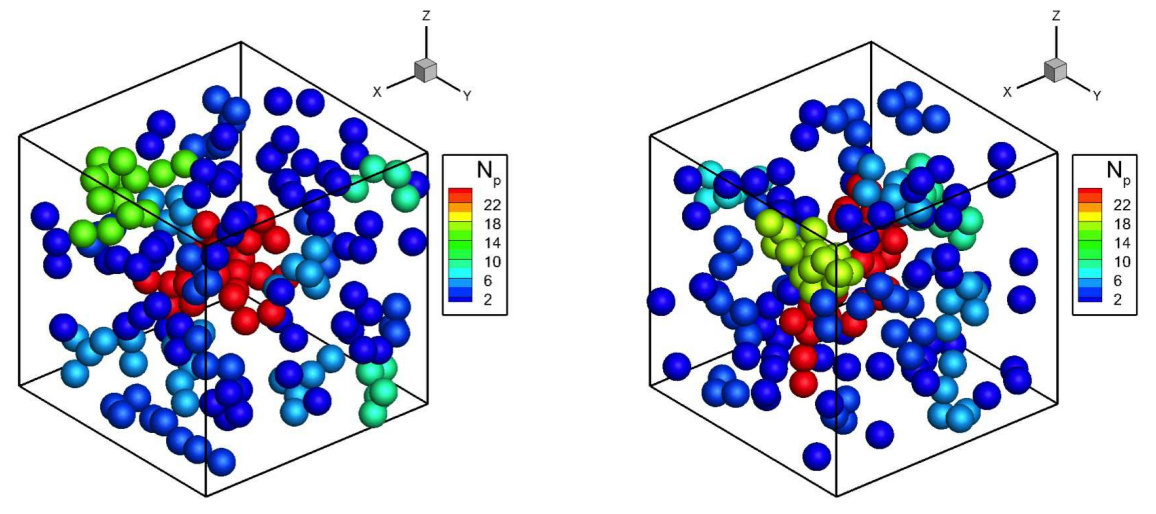

(a) $\phi=0.1$

(b) $\phi=0.1$
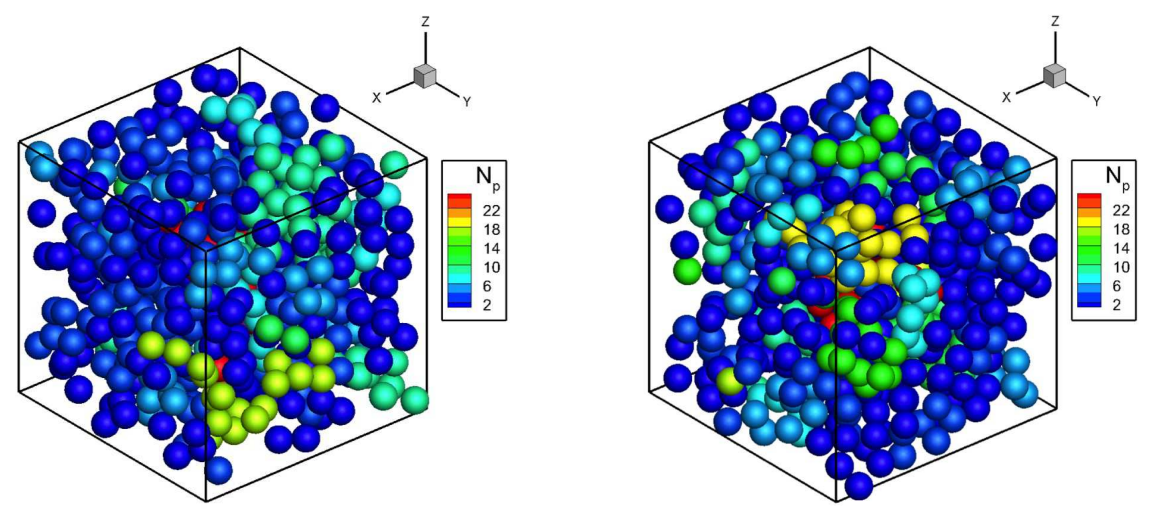

(c) $\phi=0.3$

(d) $\phi=0.3$

Figure 5: Examples of the sub-ensemble configurations for $(\mathrm{a}, \mathrm{b}) \phi=0.1$, and $(\mathrm{c}, \mathrm{d})$ $\phi=0.3$. The contour colors represent the number of particles in a cluster. 
The comparison of $g(r)$ in Fig. 4 indicates that the structures in the subensembles have the same characteristics as those in the HCG system. It should be noted that it is more practical to compare the radial distribution functions of clusters in sub-ensembles with those obtained from experiments of gas-solid flow. However, in the absence of such experimental results, we make sure that the distributions of particles in sub-ensembles are as close as to the ones in the HCG systems.

Figure 5 represents two particle sub-ensembles for $\phi=0.1$ as well as for $\phi=0.3$ where color contours indicate the number of particles in a cluster. These sub-ensembles clearly show that there exist different cluster sizes in the selected configurations. These sub-ensembles are used as particle configurations in our PR-DNS.

\section{Numerical method}

Several numerical methods have been developed for particle-resolved direct numerical simulation (PR-DNS) of fluid-solid flows (Johnson and Tezduyar, 1997; Peskin, 2002; Chen and Doolen, 1998; Prosperetti and Oguz, 2001; Feng and Michaelides, 2004). In this study, we use the particle-resolved uncontaminated-fluid reconcilable immersed boundary method (PUReIBM) of Tenneti et al. (2010). This method is shown to be accurate and numerically convergent (Garg et al., 2010b; Tenneti et al., 2011). In addition, PUReIBM has been successfully used to simulate flow over uniformly distributed particles in fixed assemblies (Tenneti et al., 2011, 2013; Mehrabadi et al., 2015) and propose a gas-particle drag law for uniform particle configurations (Tenneti et al., 2011). 
In PUReIBM, the Navier-Stokes equations are solved on a three dimensional Cartesian grid for the whole computational domain. The instantaneous conservation equations of mass and momentum are

$$
\nabla \cdot \mathbf{u}=0
$$

and

$$
\rho^{(f)} \frac{\partial \mathbf{u}}{\partial t}+\rho^{(f)} \mathbf{S}=-\mathbf{g}_{\mathrm{IBM}}+\mu^{(f)} \nabla^{2} \mathbf{u}+\mathbf{f},
$$

respectively, where $\mathbf{u}$ is the instantaneous velocity, $\mathbf{S}=\nabla \cdot(\mathbf{u u})$ is the convective term in conservative form, and $\mathbf{g}_{\mathrm{IBM}}=\nabla p$ is the pressure gradient. The no-slip and no-penetration conditions on the fluid velocity at the particle surfaces is imposed via the additional immersed boundary (IB) force term $\mathbf{f}$ in Eq. (10).

To compute the IB force, the surface of the sphere is represented by a discrete number of points called boundary points that are parametrized in spherical coordinates. Two additional sets of points, termed as exterior and interior points are generated by projecting the boundary points onto spheres of radii $r+\Delta r$ and $r-\Delta r$ respectively, with $\Delta r$ chosen to be equal to the grid spacing. In PUReIBM, the IB force is computed at the interior points so that a desired velocity $\mathbf{u}^{(k, d)}$ is obtained at the $k^{\text {th }}$ interior point. Following the direct forcing method proposed by Mohd-Yusof (1996) the IB force $\mathbf{f}^{(k)}$ at the $k^{\text {th }}$ interior point is specified to cancel the remaining terms in the momentum conservation, and to force the velocity to its desired value $\mathbf{u}^{(k, d)}$ :

$$
\mathbf{f}^{(k)}=\rho^{(f)} \frac{\mathbf{u}^{(k, d)}-\mathbf{u}^{(k, n)}}{\Delta t}+\rho^{(f)} \mathbf{S}^{(k, n)}+\mathbf{g}_{\mathrm{IBM}}^{(k, n)}-\mu^{(f)} \nabla^{2} \mathbf{u}^{(k, n)} .
$$

Note that all the terms in the above equation are evaluated at the $k^{\text {th }}$ interior point. The desired velocity $\mathbf{u}^{(k, d)}$ depends on the velocity of the particle. 
For instance, for a fixed particle the desired velocity at the interior point is equal in magnitude but opposite in direction of the fluid velocity at the corresponding exterior point so that the velocity at the boundary point is zero. The IB force so computed at all the interior points is interpolated to the neighboring grid nodes that do not include those lying in the fluid phase to get $\mathbf{f}$.

The governing equations in PUReIBM are decomposed into time-varying volumetric mean and spatio-temporal fluctuations. These quantities are simultaneously solved at each time step. The fluctuating fields are solved using a pseudo-spectral method, with the Crank-Nicolson scheme for the viscous terms, and an Adams-Bashforth scheme for the convective terms. A fractional time-stepping method that is based on Kim and Moin's approach (Kim and Moin, 1985) is used to advance the fluctuating velocity fields in time.

The salient feature of PUReIBM is that the IB forcing in PUReIBM is non-zero only inside the solid phase, and the fluid-phase is uncontaminated by the IB forcing. Therefore, the velocity and pressure in the fluid phase satisfy the unmodified Navier-Stokes equations. In addition, the hydrodynamic force experienced by a particle is computed directly from the stress tensor at the particle surface that is obtained from this uncontaminated fluid flow solution. This feature enables us to directly compare the DNS solution with any random-field theory of multiphase flow (Garg et al., 2010b; Tenneti et al., 2011), and in particular we can quantify the drag force of particles in clustered configurations. 


\section{PR-DNS results}

Before measuring the clustered particle drag force over a range of flow parameters, we need to check the grid convergence behavior of the drag force with respect to particle grid resolution. For each solid-phase volume fraction, we choose a representative particle configuration. We then perform PR-DNS of flow passing through this configuration with different grid resolutions that are defined as the number of grid points across each particle, i.e. $D_{m}=$ $d_{p} / \Delta x$. We perform these convergence tests for the mean slip Reynolds number $R e_{m}=50$ that is expected to be the upper limit for Geldart $\mathrm{A}$ particles. The grid convergence study at this $R e_{m}$ ensures that the drag force is also grid independent. The convergence behavior of the drag force for a specified particle sub-ensemble for different solid-phase volume fractions is given in Fig. 6(a), while the relative error in the drag is shown in Fig. 6. It should be noted that the relative error is the difference between the mean drag force for a given grid resolution and that obtained from the highest grid resolution normalized by the drag force of the highest grid resolution, ie. $\left|F-F_{\text {fine }}\right| / F_{\text {fine. }}$ Based on this analysis, we use a particle grid resolution of $D_{m}=30$ for $\phi=0.1$ and 0.2 , and $D_{m}=40$ for $\phi=0.3$ and 0.4 . It should be noted that the grid resolution requirement for clustered configurations are slightly higher than those used for uniform particle configurations (Tenneti et al., 2011). This is because in clustered configurations, particles are closer to each other, and therefore a finer grid resolution is needed to resolve the flow between a pair particles in a cluster.

Simulations are performed with different mean slip Reynolds numbers ranging from $R e_{m}=0.01$ to 50 and solid-phase volume fraction in the range 


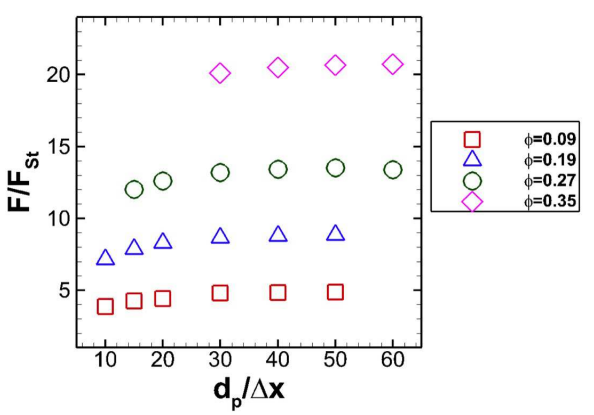

(a) $\phi=0.1$

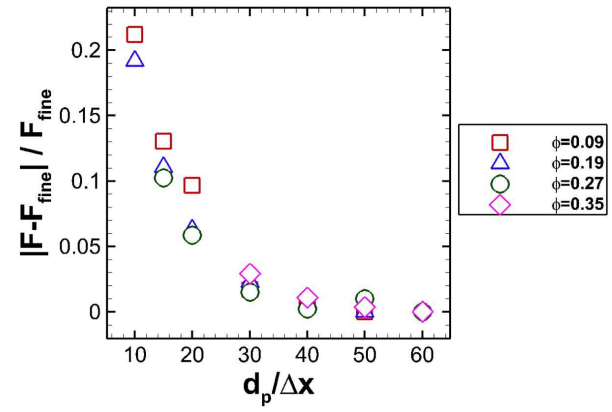

(b) $\phi=0.1$

Figure 6: (a) Grid convergence behavior of a clustered particle sub-ensemble at mean slip Reynolds number $R e_{m}=50$. (b) The relative error with respect to the highest grid resolution.

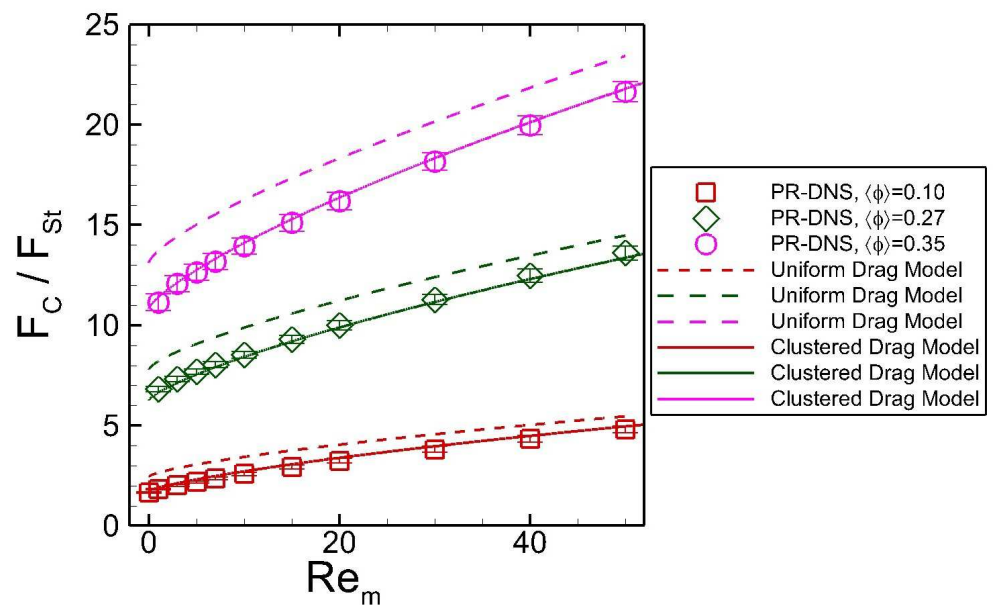

Figure 7: Mean drag force per particle in a clustered configuration normalized by the Stokes drag. Symbols represent the PR-DNS results of five sub-ensembles of particle clusters with the error bars being the $95 \%$ confidence intervals. Dashed lines show the drag law of Tenneti et al. (2011) for uniformly distributed particles in a homogeneous suspension. Solid lines represent the clustered drag law given in Eq. 17. 
Table 1: Details of cluster configurations used for PR-DNS. The columns provide in order: the mean solid-phase volume fraction, the length of the computational box to particle diameter ratio, the grid resolution across a particle, and the number of particles in the configuration. It should be noted that five independent realizations are considered for each case, and $\langle\phi\rangle$ and $N_{p}$ are the mean values obtained from these realizations.

\begin{tabular}{cccc}
\hline$\langle\phi\rangle$ & $\mathcal{L} / d_{p}$ & $d_{p} / \Delta x$ & $N_{p}$ \\
\hline 0.10 & 10 & 30 & 191 \\
0.27 & 10 & 40 & 515 \\
0.35 & 10 & 40 & 668 \\
\hline
\end{tabular}

$0.1 \leq \phi \leq 0.4$ with five realizations that correspond to difference particle configurations $(\langle\phi\rangle=0.1,0.27$ and 0.35 with $\langle\phi\rangle$ being the mean volume fraction of the five realizations). The highest mean slip Reynolds number considered in this study is 50 because Geldart A particles are relatively lighter and smaller compared to Geldart B particles. In other words, the particle Stokes number for Geldart A particles is smaller than Geldart B particles. Therefore, although the particle Reynolds number for Geldart B particle may reach as high as 300, the mean slip Reynolds number is expected to be small up to $R e_{m}=50$ for Geldart A particle. In addition, we suspect that the clusters may not survive at higher Reynolds numbers. Table 1 provides details of the cases simulated in this study. Quantification of the mean drag force per particle normalized by the Stokes drag is shown in Fig. 7. The comparison of the PR-DNS results for the clustered cases with the uniform drag model of Tenneti et al. (2011) indicates that the mean drag reduces in clustered configurations for the range of $\langle\phi\rangle$ and $R e_{m}$ considered in the current study. The relative amount of drag reduction compared with the 
uniform drag model of Tenneti et al. (2011) is shown in Fig.8. This figure indicates the maximum reduction up to about $35 \%$ for $\langle\phi\rangle=0.1$ in Stokes flow when compared to the uniform drag model of Tenneti et al. (2011).

It is observed in Fig. 8 that the amount of drag reduction decreases with increase of mean slip Reynolds number. The attenuation of drag reduction with Reynolds number is associated with the reduction of the Brinkman screening length as the Reynolds number increases. The Brinkman screening length is equivalent to the integral length scale associated with the Eulerian two-point covariance of velocity fluctuations (Koch et al., 1998). Mehrabadi et al. (2015) showed that the integral length scale decreases with increasing mean slip Reynolds number. Therefore, increase of the Reynolds number causes the flow velocity to decorrelate over shorter length scales that leads to smaller drag reduction.

Figure 8 reveals that the amount of drag reduction is higher for low solidphase volume fraction $\langle\phi\rangle=0.1$ compared with higher volume fractions. It also shows that the level of drag reduction for the cases with volume fraction $\langle\phi\rangle=0.27$ and 0.35 are the same. This leveling off of the drag reduction behavior with respect to solid-phase volume fraction can be explained by a closer look at clustering metrics.

Several methods for characterizing the level of clustering have been proposed. One approach is to obtain the probability density function (PDF) of Voronoi cell volumes (Monchaux et al., 2010). In this method, Voronoi cells are constructed around the center of each particle such that they contain only a single particle. This construction of Voronoi cells leads to appearance of small volume cells in regions where particles are very close to each other, 


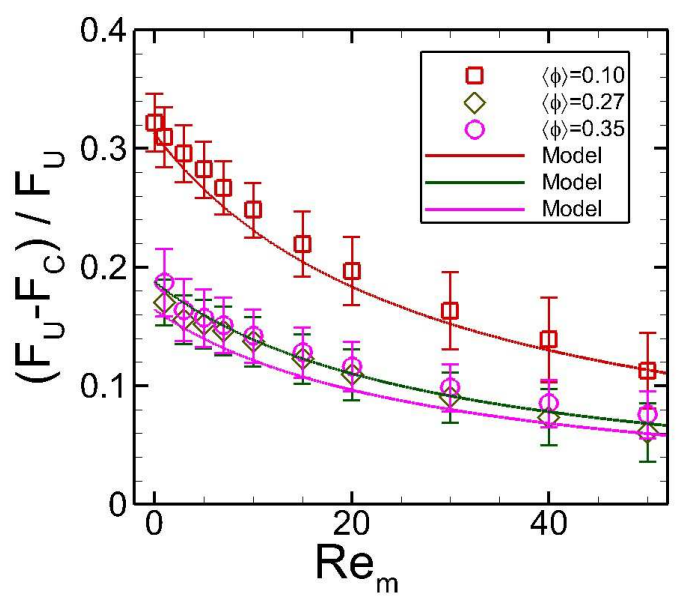

Figure 8: Symbols show the relative amount of drag reduction from PR-DNS of clustered configurations when compared with the uniform drag model of Tenneti et al. (2011). The error bars show the $95 \%$ confidence intervals obtained from five independent subensembles. The solid lines represent the drag reduction model given by Eq. 16 .

in contrast to the void regions where the volume of Voronoi cells is relatively large. Comparison of the PDF of these Voronoi cell volumes when compared to that obtained from the case with uniformly distributed particles indicates the level of clustering. The disadvantage with using Voronoi cell volumes as a metric of clustering is that it is difficult to construct a transport equation for this quantity for use in two-fluid multiphase CFD simulations.

Another approach is excess two-body entropy which is based on the radial distribution function of particle configuration (Baranyai and Evans, 1989). Recall that the radial distribution function $g(r)$ provides a two-point statistics about the location of particles with respect to each other. Therefore, configurational information of a particle assembly is embedded in the radial distribution function. The excess entropy is computed from the following 
expression:

$$
S_{\text {excess }}^{(2)}=-\frac{1}{2} \rho \int 4 \pi r^{2} g(r) \ln (g(r)) d r+\frac{1}{2} \rho \int 4 \pi r^{2}(g(r)-1) d r .
$$

If a particle configuration has a Poisson distribution, then the corresponding radial distribution function is unity for all separations. It can be easily shown that for such a $g(r)$, the excess two-body entropy from Eq. 12 is zero. However, if particle clusters exist, the $g(r)$ deviates from unity, as shown in Fig. 4, which in turn leads to a non-zero excess two-body entropy. In this case, the value of $S_{\text {excess }}^{(2)}$ determines how far a configuration is from the uniformly distributed state.

Although the above method provides an appropriate metric for characterizing the level of particle clustering when particle locations are changed, it cannot be generalized to a case where the number of particle change from one realization to another. Usually, the mean volume fraction for a homogeneous configuration is defined from averaging $\phi$ over different realizations. Therefore, volume fraction which is related to the number of particles in a volume may change from one realization to another. In other words, the number of particles is itself a random variable that may be different between two realizations. This randomness in the number of particles is not considered in Eq. 12. However, a more general approach that allows randomness in the number of particles in addition to the randomness in particle configuration is the configurational entropy of a point process method (Murphy and Subramaniam, 2016b).

In this method an entropy for the configuration can be computed by reconstructing the probability distribution function of particle number density in sub-volumes taken from a simulation. If we consider the one-particle den- 
sity function, constructed from a measurement volume, for a grand canonical ensemble, that is with a variable number of particles, we obtain the following expression:

$$
f\left(\mathbf{x}, \mathbf{v}, t ; V_{m}\right)=\sum_{k} P\left(k ; V_{m}\right) f^{(k)}\left(\mathbf{x}, \mathbf{v}, t ; V_{m}\right)
$$

The probability mass function $P\left(k ; V_{m}\right)$ denotes the probability that there are $k$ particles in a realization of the point process in sub-volume $V_{m}$. In the above expression $f^{(k)}\left(\mathbf{x}, \mathbf{v}, t ; V_{m}\right)$ is the one-particle distribution function of a particle assembly defined over the position and velocity sample space for a given sub-volume $V_{m}$ that includes $k$ particles (Subramaniam, 2000). We want to find the entropy of this probability mass function, constructed from a simulation. It has been shown that the entropy of a particle configuration has the following form (Subramaniam, 2014):

$$
S_{n}=-\sum_{k=0}^{\infty} P\left(k ; V_{m}\right) \ln \left(P\left(k ; V_{m}\right)\right) .
$$

The salient feature of this method is that although it may require more computational time compared to the PDF of Voronoi cells method, it is derived from a mathematical perspective explaining a physical observation. Therefore, it is possible to derive a transport equation for the configurational entropy compared to the PDF of Voronoi cells method. In addition, the configurational entropy method is more general compared to the excess twobody entropy method explained earlier since configurational entropy method allows variation in the number of particles as well.

We select the configurational entropy approach to compare the entropy of the grand canonical ensemble at two different states, for instance at uniformly 


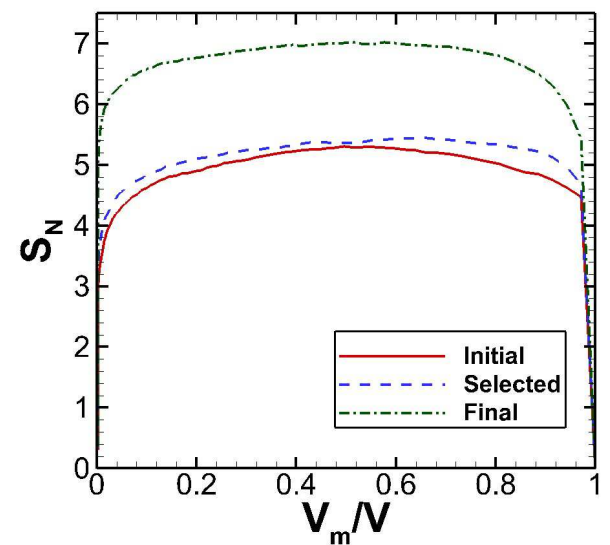

(a) $\phi=0.1$

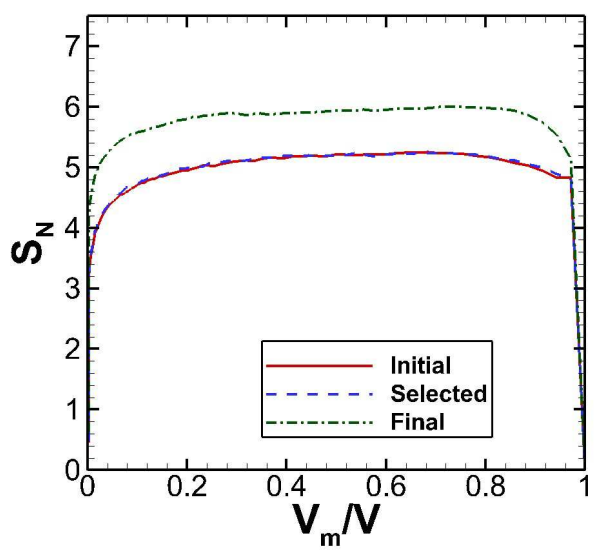

(c) $\phi=0.3$

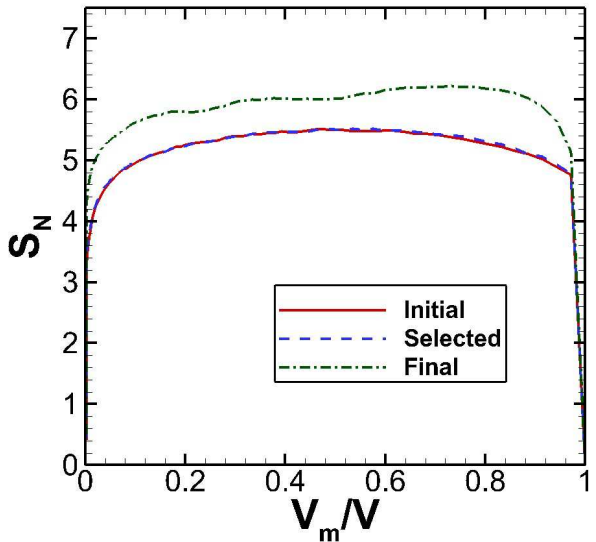

(b) $\phi=0.2$

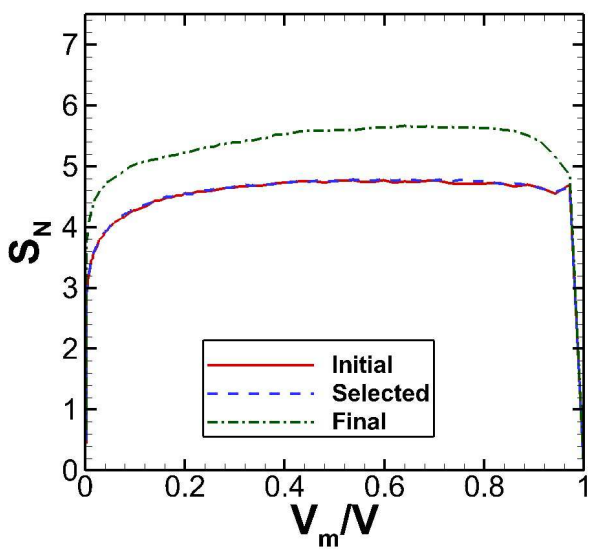

(d) $\phi=0.4$

Figure 9: Configurational entropy quantification for homogeneous cooling gas simulations as a function of measurement volume $V_{m}$ normalized by the volume of the system $V$. These quantifications has been performed for the initial condition where particles are uniformly distributed (presented with solid lines), when sub-ensemble clustered configurations are extracted for PR-DNS (shown in dashed lines), and the final state of HCG when all particles form a single giant cluster in the computational box (shown with dash-dot lines). 
distributed and clustered states, in order to measure the level of clustering in the configuration. Quantification of the configurational entropy given by Eq. 14 has been performed for the HCG systems at (i) the initial state where particle are uniformly distributed, (ii) the time the cluster-containing subensembles have been extracted for the PR-DNS in this study, and (iii) at the final time of HCG simulation when all particles in the computational box form a giant cluster. Figure 9 shows that the difference in configurational entropy between the initial state and the time when PR-DNS sub-ensembles are extracted is significant for $\langle\phi\rangle=0.1$. However, the difference in the configurational entropy between the two states for higher volume fractions is not noticeable. This indicates that the configurational changes due to the presence of particle clusters are higher for low solid-phase volume fraction since particles are allowed to rearrange more in microscale, and form simultaneous clustered and void regions. Presence of these regions in configurations at low solid-phase volume fraction substantially influences the flow hydrodynamics, and therefore higher drag reduction is achieved when compared with high solid-phase volume fraction cases. This point can also be confirmed by comparing the amount of entropy difference between the initial and selected states which seems to be relatively greater for $\langle\phi\rangle=0.1$, when compared with higher volume fractions for which the difference between the initial and selected states are very close.

\section{Clustered drag model}

For the purpose of formulating a clustered drag law, we assume that once particle clusters are formed, they match the distribution provided by 
Cocco et al. (2010). In the absence of further evidence for the evolution of cluster formation and breakup and also cluster metrics at different operating conditions, we assume that the distribution provided by Cocco et al. (2010) is a valid representation for particle clusters in a fluidized bed. This allows us to provide a drag model from our PR-DNS results of homogeneous gas-solid flow when the cluster size distribution in PR-DNS approximately matches the one reported by Cocco et al. (2010). Therefore, this clustered drag model would represent the interphase mean momentum transfer when the maximum clustering state exists in the suspension.

Figure 8 indicates that the correlation for drag reduction can be a function of the solid-phase volume fraction and the mean slip Reynolds number:

$$
G\left(\phi, R e_{m}\right)=\frac{F_{U}\left(\phi, R e_{m}\right)-F_{C}\left(\phi, R e_{m}\right)}{F_{U}\left(\phi, R e_{m}\right)},
$$

where $F_{C}$ and $F_{U}$ are, respectively, the clustered and uniform drag models. Based on the drag reduction quantification in Fig. 8 and physical considerations, the following constraints should be imposed on $G\left(\phi, R e_{m}\right)$ :

1. The function should be finite in Stokes flow $\left(R e_{m} \rightarrow 0\right)$ because the maximum drag reduction appears at Stokes flow

2. The function should tend to zero at high Reynolds number flow $\left(R e_{m} \rightarrow\right.$ $\infty)$ due to the fact that the Brinkman screening length monotonically decreases with Reynolds number and the flow seems to feel the particles as isolated sphere rather than as in clusters

3. The function should monotonically increase with the decrease of volume fraction, reaching an upper limit of unity, i.e.

$$
\lim _{\phi \rightarrow 0} G\left(\phi, R e_{m}\right)=1 .
$$


Considering the above constraints, we propose a model for drag reduction with the following form

$$
G\left(\phi, R e_{m}\right)=\left(\frac{a}{1+b R e_{m}}\right)\left(1-\exp \left(-c \phi^{d}\right)\right)
$$

which provides a good match to our PR-DNS results as shown in Fig. 8 for the range of flow parameters considered in this study. The model constants in the above expression are $a=1.16 \times 10^{1}, b=3.52 \times 10^{-2}, c=8.25 \times 10^{-3}$, $d=-5.19 \times 10^{-1}$.

It is possible to rearrange the terms in Eq. 15 to obtain the clustered drag model with respect the the uniform drag law and the drag reduction model, given as

$$
F_{C}\left(\phi, R e_{m}\right)=\left\{1-G\left(\phi, R e_{m}\right)\right\} F_{U}\left(\phi, R e_{m}\right),
$$

where the drag model of Tenneti et al. (2011) for $F_{U}$ and Eq. 16 for $G$ are used. This clustered drag model has been compared with PR-DNS results of cluster-containing sub-ensembles in Fig. 7, and indicates a good agreement between the model and simulations for the range of parameters considered in this study. As mentioned earlier, it is assumed that this drag model represents the mean momentum transfer when the system is at the maximum state of clustering.

It should be noted that cluster statistics are subject to change with respect to flow regime, particle properties, and device geometry. Clusters statistics may depend on many other quantities and they may not be universal. However, even in clusters with very large number of particles, we expect that for particles deeper than those in the surface layer the average force experienced by a single particle will not change significantly with the number of particles 
in the cluster. The average force on the entire clustered particle configuration also necessarily depend on cluster statistics whose universality is in question. In addition, it is not clear from the literature as to how the interplay between hydrodynamic and cohesive forces gives rise to the formation of clusters over a wide range of flow conditions and particle properties (McMillan et al., 2013). Therefore, a priori prediction of cluster statistics given flow conditions and particle properties is still an unanswered question. Therefore, the drag model presented in this study is not a generalized model that is applicable to all clustering systems. However, we believe that the presented model is applicable to the polyethylene and FCC particles that are used in the experimental setup of Cocco et al. (2010). Since the proposed clustered drag model is different from the uniform drag model, the transition between the uniform and clustered drag models is discussed in the next section.

\section{Drag model: transition between uniform and clustered drag laws}

In general, the drag force on a clustered particle configuration, depends on cluster dynamics as well as clustering metrics. It is known that the cluster dynamics depend on gas-particle and particle-particle interactions. However, details of the dynamics that lead to formation and breakup of particle clusters is not yet well understood. Characterization of clusters at microscopic scales requires detailed information about particle locations that may not be available in coarse grained or two-fluid theory approaches. The solution to this discrepancy is either to develop a transport equation for clustering metrics that are closed with respect to flow-transported quantities, or to consider particle clusters with presumed characteristics and corresponding metrics. 
As a starting point, we assume that the balance of processes determining the formation and break-up of clusters is at a steady state. Therefore, no significant changes in the state of clustering in a particle configuration occur. Also in the absence of adequate knowledge about the mechanism of hydrodynamic interactions that lead to formation of particle clusters as of those observed by Capecelatro et al. (2014), we assume that for Geldart A particle the major driving mechanism for formation of clusters is particle cohesion. The balance between production of kinetic energy from the mean flow, and dissipation of energy would then produce a unique granular temperature and distribution of cluster sizes for a given attractive interparticle potential (such as cohesion, electrostatic, van der Waals forces). As mentioned earlier, the ratio between the potential energy and fluctuating kinetic energy is characterized by the $\mathrm{Ha}$ parameter given by Eq. 7. Therefore, the tentative form of the general drag model can be described as:

$$
F=f\left(\phi, R e_{m}, H a\right)
$$

The $H a$ parameter can be dynamically computed in each computational cell of a CFD simulation using the two-fluid approach or CFD-DEM approach. The $H a$ parameter is obtained by knowing the energy associated with the attractive forces between the particles (characterized by Hamaker constant, particle diameter, minimum allowed particle separation) and the granular temperature in each cell. Once $H a$ exceeds a critical value, then particle clusters form, otherwise the configuration remains uniform.

The limit $\mathrm{Ha} \rightarrow 0$ is reached when either the particle granular temperature is infinite or there is no attraction among particles, and in that limit the particles are uniformly distributed inside of a CFD cell. Therefore, a uni- 
form drag law should be used in this limit. In contrast, the limit $H a \rightarrow \infty$ is achieved when the particles are strongly attracted towards each other, or the granular temperature tends to zero. In these circumstances, a clustered drag model should be used.

Switching back and forth between the uniform and clustered drag laws in a simulation may lead to numerical instabilities. Therefore, it is preferable to use a smooth transition between the two states. Although this transition can depend on several flow parameters as well as particle properties, currently we only consider the transition dependence on the $H a$ parameter. The mean drag force on a configuration is specified as a weighted sum of the two limiting drag laws in the following form:

$$
F=f\left(\phi, R e_{m}, H a\right)=\{1-h(H a)\} F_{U}\left(\phi, R e_{m}\right)+h(H a) F_{C}\left(\phi, R e_{m}\right),
$$

where $h(H a)$ is the smooth function used to switch between the uniform and clustered drag models. Since particle clusters dynamically form and break up, $h$ should be determined based on the dynamics of the system. However, because of the lack of any information about the behavior of the transition between the uniform and clustered drag forces, we limit ourselves to a monotonically increasing function with respect to $H a$ with the following form:

$$
h(H a)=\frac{1}{2}+\frac{1}{\pi} \arctan \left(\frac{H a-H a_{0}}{\varepsilon}\right) .
$$

In the above expression, $H a_{0}$ determines the critical value of $H a$ when the switching between the uniform and clustered drag models takes place. Additionally, the parameter $\varepsilon$ determines the slope of switching between the two states. This family of curves asymptotes to a Heaviside function in the 


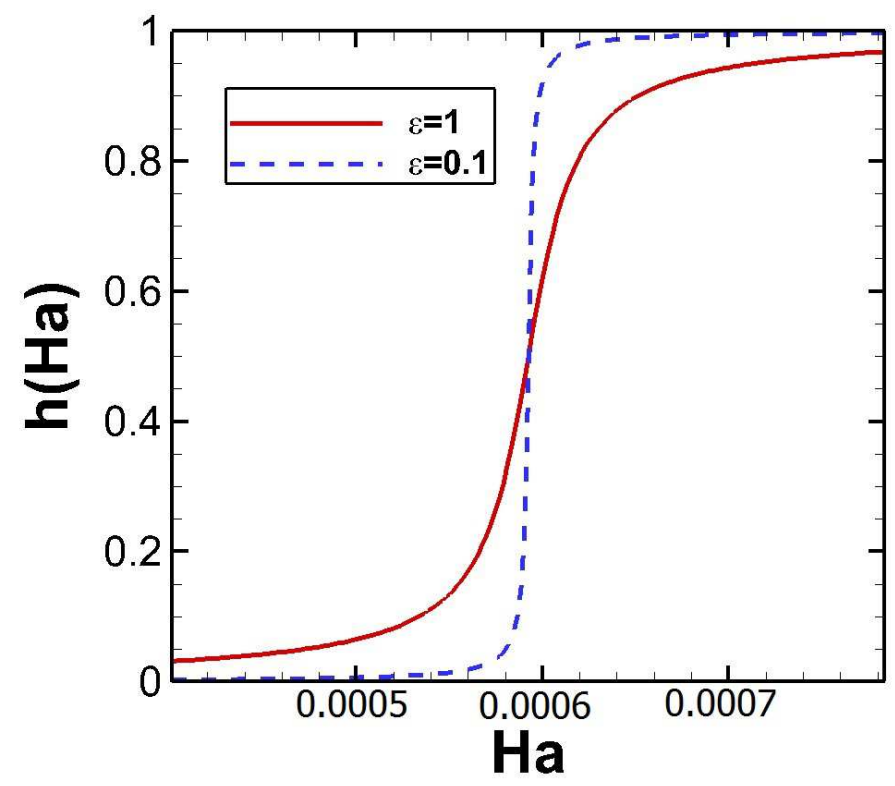

Figure 10: The shape of $H a$ function given by Eq. 20 .

limit of $\varepsilon \rightarrow 0$. The parameter $\varepsilon$ is a model constant that should be specified based on model testing.

The $h$ function given in Eq. 20 is anti-symmetric about $H a_{0}$. The value for this parameter can be either determined by experimental analysis, the Type 1 PR-DNS of gas-solid flow explained in Section 2, or model testing in CFD simulations. For instance to show how we can estimate $H a_{0}$ based on our PR-DNS results, we consider a case with solid-phase volume fraction $\phi=0.1$ and particle diameter $d_{p}=50 \mu \mathrm{m}$ suspended by a mean flow with mean slip Reynolds number up to 100. For this case, the particle granular temperature is approximately in the range of $0.064 \mathrm{~m}^{2} / \mathrm{s}^{2}$ to $2.728 \mathrm{~m}^{2} / \mathrm{s}^{2}$ (Tenneti et al., 2016). If we assume that the Hamaker constant is $A=10^{-19} \mathrm{~J}$, the solidphase density ratio is 400 , and the minimum particle separation is $d_{0}=2$ Angstrom, then the minimum and maximum values of $\mathrm{Ha}$ for the particles 
suspended in an air flow would be $H a_{\min }=3 \times 10^{-5}$ and $H a_{\max }=1.2 \times 10^{-3}$, respectively. The $H a_{0}$ is then defined as the average value of these two limits, that is

$$
H a_{0}=\frac{H a_{\max }+H a_{\min }}{2}=6 \times 10^{-4} .
$$

This $H a_{0}$ value is chosen for representing the $h(H a)$ function in Fig. 10. Nevertheless, both $H a_{0}$ and $\varepsilon$ are model constants and should be used to tune the model for CFD simulations. These tuning parameters are problem-sensitive, and should be set for each particular application. The tuning process can be preformed by simulating a fluidized bed for which the experimental data are also available. In the simulations, the tuning variables $H a_{0}$ and $\varepsilon$ should be such that the numerical results match the experimental findings, such as the pressure drop or solid-phase volume fraction profiles along the bed. In order to accelerate the tuning process, an appropriate optimization algorithm can also be employed. Once the model parameters are tuned, the model can be used in CFD simulation of other geometries and applications.

It should be highlighted that in this work we chose the $\mathrm{Ha}$ parameter based on the energy associated in the particle attractive forces and characteristic kinetic energy of a pair of particles as a metric to determine existence of particle clusters in the proposed model. Although this parameter does not account for the formation of clusters due to solely hydrodynamics interactions, it is a good candidate for cluster detection in Geldart A gas-solid flows where the main mechanism of clustering is cohesive forces. In addition, in the absence of experimental results for realizations of particle clusters in gas-solid flow, we used HCG simulations to produce clusters that match in size to those reported by (Cocco et al., 2010). Once experimental results 
for clusters of particles in gas-solid flows for difference flow parameters and particle properties become available, then the clustered drag model proposed by Eq. 17 may be revisited for better predictions in CFD applications.

\section{Conclusion}

In this work, we used PR-DNS to quantify the mean gas-particle drag in gas-solid suspensions where the particle configurations contain particle clusters. These simulations are performed in homogeneous flow setup in order to isolate the effect of interphase interaction on particle clusters. In our PR-DNS, we used clustered configurations that approximately matched the statistics of experiments, while earlier works used deterministic approaches to generate particle configurations for PR-DNS. We extracted the clustered particle configurations from DEM simulation of a HCG with inelastic and cohesive particles in the absence of interstitial fluid. The selected clustercontaining sub-ensembles were selected to match the cluster distribution provided by the experiment (Cocco et al., 2010). PR-DNS of flow over these sub-ensembles indicates that existence of particle clusters lead to drag reduction compared with configurations that contain uniformly distributed particles at the same flow condition. Our PR-DNS results indicate that the maximum drag reduction appears at Stokes flow. The attenuation of drag reduction with Reynolds number is associated with reduction of the Brinkman screening length. Therefore, an increase in the mean slip Reynolds number reduces the spatial extent of the flow disturbance caused by particles. This makes the flow see particles as individual entities rather than a cluster, which leads to attenuation of drag reduction. Our results also indicate that 
the drag reduction attenuates with increase of solid phase volume fraction. In order to understand the reason for this attenuation, we introduced a cluster metric to measure configurational entropy for a particle assembly. Our analysis indicates that the changes in configurational entropy of particles in dilute regimes is higher compared with dense suspensions. Therefore, particle configurations in the dilute regimes change significantly when particles form clusters as compared to dense suspensions. Clustering in turn leads to higher drag reduction for dilute regimes.

We have used our PR-DNS data to propose a drag law for clustered configurations for the range of flow parameters considered in this study, that is $0.1 \leq\langle\phi\rangle \leq 0.35$ and $0.01 \leq R e_{m} \leq 50$. This drag model has been proposed based on the amount of drag reduction when compared with the uniform drag model of Tenneti et al. (2011). We have also provided a smooth transition between the uniform and clustered drag models for stability of CFD simulations by means of using a weighting function. This function has two adjusting parameters that can be determined by comparing CFD predictions with experimental results, such as solid-phase volume fraction profile or pressure drop along a fluidized bed riser. The tuned model then can be used for CFD simulations of complex geometries and engineering applications.

\section{Acknowledgments}

This work is partially supported by Department of Energy grant DEFC26-07NT43098 and DE-FE0007260 through the National Energy Technology Laboratory (NETL). We would also like to acknowledge the National 
Science Foundation for partial support from award CBET 1134500.

\section{Appendix A. Cohesive to contact and cohesive to hydrodynamic force ratios}

In this appendix, both cohesive to contact and cohesive to hydrodynamic force ratios are reported. It should be noted that for simulations of fixed particle clusters that are reported in this work, the ratio of the cohesive force to the contact force is not relevant because particles do not move or undergo collisions. It is relevant for the real problem, and there is a critical value of the granular temperature below which particles will remain in clusters, and above which they do not. Also it should be noted that the causes and mechanism of clustering in fluidized beds and risers is yet unknown, and the relative importance of hydrodynamic and interparticle forces in the formation and persistence of clusters is not established (McMillan et al., 2013). We now present the two force ratios. Since the van der Waals potential is given by Eq. 1 in the manuscript, the cohesive force is obtained by

$$
F_{c o h}(r)=-\frac{d}{d r} U_{c o h}(r)=-\frac{A d_{p}}{24\left(r-d_{p}+d_{0}\right)^{2}} .
$$

The maximum value of the cohesive force occurs when the separation between the centers of the two particle is $d_{p}$. Therefore, the maximum magnitude of the cohesive force is:

$$
\max \left[F_{c o h}(r)\right]=F_{c o h}^{(\max )}=\frac{A d_{p}}{24 d_{0}^{2}} .
$$

This value remains constant during the collision process, since the separation $r$ has already reached the inner cut-off distance $d_{p}$. This maximum force is often encapsulated in the modified Bond number 


$$
B o^{\star}=\frac{A}{24 d_{0}^{2} k},
$$

which is the cohesive force at contact normalized by a characteristic spring force. The modified Bond number also indicates the resting overlap between two spheres in contact, $B o^{\star}=\delta_{c o h} / d_{p}$. A similar scaling can be found by appropriately non-dimensionalization of equation 3 in the manuscript. Dividing both sides by the characteristic scale for potential energy in the spring and taking a square root one obtains

$$
\sqrt{\frac{m v^{2}}{k d_{p}^{2}}}=\frac{1}{\sqrt{k^{\star}}}=\delta / d_{p},
$$

where $\delta$ is the maximum overlap during a collision between non-dissipative spheres. The velocity scale chosen here is given in terms of temperature $v=\sqrt{T}$. The ratio of the overlaps $\delta_{c o h}$ and $\delta$ then gives a ratio between cohesive and contact forces

$$
\frac{F_{\text {coh }}^{(\max )}}{F_{\text {cont }}^{(\max )}}=B o^{\star} \sqrt{k^{\star}} .
$$

To find characteristic values for simulation we use values typical of both Plascoat $^{T M} 571$ (PPA571) used in Cocco et al. (2010) and also FCC particles. For PPA571 particles we have found the properties: $d_{p}=70$ microns, $d_{0}=2$ Angstroms, $A=10^{-19}$ Joules, $\rho=960 \mathrm{~kg} / \mathrm{m}^{3}, E=14 G P a, \nu=0.3$. While FCC particles are slightly denser and stiffer than PPA571 particles: $d_{p}=70$ microns, $d_{0}=2$ Angstroms, $A=10^{-19}$ Joules, $\rho=1400 \mathrm{~kg} / \mathrm{m}^{3}, E=35 G P a$, $\nu=0.3$. Here Poisson's ratio $\nu$ and the Hamaker constant $A$ of PPA571 and FCC particles have been assumed in relation to other materials. If we seek to 


\begin{tabular}{lcccc}
\hline$R e$ & $k_{F C C}^{\star}$ & $B o^{\star} \sqrt{k^{\star}} F C C$ & $k_{P P A 571}^{\star}$ & $B 0^{\star} \sqrt{k^{\star}} P P A 571$ \\
\hline 1 & $5.57 \times 10^{10}$ & $3.42 \times 10^{-2}$ & $3.25 \times 10^{10}$ & $6.55 \times 10^{-1}$ \\
20 & $1.39 \times 10^{8}$ & $1.71 \times 10^{-3}$ & $8.16 \times 10^{7}$ & $3.28 \times 10^{-3}$ \\
\hline
\end{tabular}

Table A.2: The values of non-dimensional parameters for different Reynolds numbers.

match the $k^{\star}$, defined as in A.5, for a corresponding Hertzian spring model, the spring stiffness is given as

$$
k=\frac{4}{15} \frac{E}{\left(1-\nu^{2}\right)} d_{p} .
$$

The stiffness is found to be for $2.8 \times 10^{5} \mathrm{~kg} / \mathrm{s}^{2}$ and $7.2 \times 10^{5} \mathrm{~kg} / \mathrm{s}^{2}$ PPA571 and FCC particles, respectively. For a Reynolds numbers of 1 and 20 with the properties of air at $\left(\rho_{f}=1.177 \mathrm{~kg} / \mathrm{m}^{3}\right.$ and $\left.\mu_{f}=1.846 \times 10^{-5} \mathrm{~kg} / \mathrm{ms}\right)$, the expected particle temperatures are $T \approx 5.02 \times 10^{-4} \mathrm{~m}^{2} / \mathrm{s}^{2}$ and $T \approx 0.2$ $\mathrm{m}^{2} / \mathrm{s}^{2}$. The ratio $T /\left|\left\langle\mathbf{W}^{2}\right\rangle\right|=0.01$ given on page 13 of the manuscript was used. We stress that the mean slip is unknown apriori. These FCC particles are small, and should not experience especially large slip numbers due to their small size. The modified Bond number for PPA571 and FCC systems are $B o^{\star}=3.63 \times 10^{-7}$ and $B o^{\star}=1.45 \times 10^{-7}$, respectively. The values for $k^{\star}$ and $B o^{\star} \sqrt{k^{\star}}$ are summarized in Table A.2.

For all cases, the cohesive force is smaller than the maximum force experienced during impact. However, it is important to note that clustering when due to particle collisions, is not controlled by a balance of forces. In fact, for all non-dimensional overlaps $\delta / d_{p}$ less than $B o^{\star}$, the cohesion force dominates. Rather, it is the dissipation during the collision that leads to particle aggregation, which is also enhanced by cohesion.

In a similar way, the ratio of the maximum cohesive force to the drag force 
can be computed. The Stokes drag force is selected as the representative of the hydrodynamic force, and many drag laws have been cast using premultiplying drag factors that correct the Stokes drag. In addition, the Stokes drag can be easily related to mean drag force at a given solid-phase volume fraction and Reynolds number. The drag force that we will consider is given by

$$
F_{d r a g}=C\left(\phi, R e_{m}\right) F_{S t}=3 C \pi d_{p} \mu_{f}|\langle\mathbf{W}\rangle|,
$$

where $\langle\mathbf{W}\rangle, C, \phi$ and $R e_{m}$ are the slip velocity, drag factor, solids volume fraction, and slip Reynolds number. Now the cohesive to Stokes drag ratio can be obtained by dividing Eq. A.2 by Eq. A.7, which is given in terms of both dimensional and non-dimensional terms

$$
\frac{F_{c o h}^{(\max )}}{F_{S t}}=\frac{A}{72 C \pi d_{0}^{2} \mu_{f}|\langle\mathbf{W}\rangle|}=\frac{S t}{C}\left(\frac{R e_{T}}{R e_{m}}\right)^{2} B o^{\star} k^{\star} .
$$

Here $S t$ and $R e_{T}$ are the mean Stokes number and the Reynolds number from the granular temperature. Note that this combination of nondimensional parameters does depend on mass or stiffness of particles. For the case of Stokes flow and Plascoat ${ }^{T M} 571$ (PPA571) particles, i.e. $R e_{m}=1$ and $C=1$, the ratio is equal to 5,340 . Therefore, in the Stokes flow regime, the cohesive force is dominant compared with the mean hydrodynamic drag. However, as the hydrodynamic force increases with the increase of solidphase volume fraction and mean slip Reynolds number, or the cohesion force is made smaller due to surface roughness, the hydrodynamic force can come to outweigh the cohesive force.

It should also be noted that the lubrication force may be a better representative of the hydrodynamic force during a particle collision. Lin and 
Lin (2013) have shown that the ratio of the lubrication force to the Stokes drag could be as high as 100 when a particle is in the vicinity of a solid wall. By assuming that this number is approximately valid for a pair of particles, then the cohesive to hydrodynamic force ratio given above reduces about two orders of magnitude when the two particles are very close to each other.

\section{References}

Baranyai, A., Evans, D. J., Oct 1989. Direct entropy calculation from computer simulation of liquids. Phys. Rev. A 40, 3817-3822.

Beetstra, R., van der Hoef, M., Kuipers, J., 2006. A lattice-boltzmann simulation study of the drag coefficient of clusters of spheres. Computers and Fluids 35 (8-9), 966 - 970, proceedings of the First International Conference for Mesoscopic Methods in Engineering and Science.

Beetstra, R., van der Hoef, M. A., Kuipers, J. A. M., 2007. Drag force of intermediate Reynolds number flows past mono- and bidisperse arrays of spheres. AIChEJ 53, 489.

Capecelatro, J., Desjardins, O., Fox, R. O., 5 2014. Numerical study of collisional particle dynamics in cluster-induced turbulence. Journal of Fluid Mechanics 747.

Chen, S., Doolen, G. D., 1998. Lattice boltzmann method for fluid flows. Annual Review of Fluid Mechanics 30 (1), 329-364.

Chew, J. W., Hays, R., Findlay, J. G., Knowlton, T. M., Karri, S. R., Cocco, R. A., Hrenya, C. M., 2012. Cluster characteristics of geldart group b 
particles in a pilot-scale CFB riser. I. monodisperse systems. Chemical Engineering Science 68 (1), 72-81.

Cocco, R., Shaffer, F., Hays, R., Reddy Karri, S., Knowlton, T., 2010. Particle clusters in and above fluidized beds. Powder Technology 203 (1), 3-11.

Cundall, P. A., Strack, O. D. L., 1978. The Distinct Element Method as a Tool for Research in Granular Media. Tech. Rep. NSF Grant ENG7620711, National Science Foundation.

Derjaguin, B., Muller, V., Toporov, Y., 1975. Effect of contact deformations on the adhesion of particles. Journal of Colloid and Interface Science 53 (2), 314-326.

Ergun, S., 1952. Fluid flow through packed columns. Chem. Eng. Prog. 48, 89-94.

Fan, R., Marchisio, D. L., Fox, R. O., 2004. Application of the direct quadrature method of moments to polydisperse gas-solid fluidized beds. Powder Technology 139 (1), 7 - 20.

Feng, Z. G., Michaelides, E. E., 2004. The immersed boundary-lattice boltzmann method for solving fluid-particles interaction problems. Journal of Computational Physics 195 (2), 602-628.

Gao, J., Chang, J., Xu, C., Lan, X., Yang, Y., 2008. CFD simulation of gas solid flow in FCC strippers. Chemical Engineering Science 63 (7), 1827 1841. 
Garg, R., Shahnam, M., Huckaby, E. D., 2010a. Continuum simulations of co2 capture by dry regenerable potassium based sorbents. In: in ICMF. Tampa, FL.

Garg, R., Tenneti, S., Mohd-Yusof, J., Subramaniam, S., 2010b. Direct numerical simulation of gas-solids flow based on the immersed boundary method. In: Pannala, S., Syamlal, M., O'Brien, T. J. (Eds.), Computational Gas-Solids Flows and Reacting Systems: Theory, Methods and Practice. IGI Global.

Gokaltun, S., Varona, J., Awwad, A., Roelant, D., 2012. Detection of particle clustering in gassolid systems using shadow-sizing technique. Powder Technology 220, 98-103.

Harris, A., Davidson, J., Thorpe, R., 2002. The prediction of particle cluster properties in the near wall region of a vertical riser (200157). Powder Technology 127 (2), 128 - 143.

Hill, R. J., Koch, D. L., Ladd, A. J. C., 2001a. The first effects of fluid inertia on flows in ordered and random arrays of spheres. J. Fluid Mech. $448,213-241$.

Hill, R. J., Koch, D. L., Ladd, A. J. C., 2001b. Moderate-Reynolds-number flows in ordered and random arrays of spheres. J. Fluid Mech. 448, 243278.

Johnson, A. A., Tezduyar, T. E., 1997. 3D simulation of fluid-particle interactions with the number of particles reaching 100. Computer Methods in Applied Mechanics and Engineering 145 (3-4), 301-321. 
Kim, J., Moin, P., 1985. Application of a fractional-step method to incompressible Navier-Stokes equations. Journal of Computational Physics 59, 308-323.

Koch, D. L., Hill, R. J., Sangani, A. S., 1998. Brinkman screening and the covariance of the fluid velocity in fixed beds. Physics of Fluids 10 (12), $3035-3037$.

Kuwabara, G., Kono, K., 1987. Restitution coefficient in a collision between two spheres. Japanese Journal of Applied Physics 36, 1230.

Li, J., Cheng, C., Zhang, Z., Yuan, J., Nemet, A., Fett, F. N., 1999. The EMMS model its application, development and updated concepts. Chemical Engineering Science 54 (22), 5409 - 5425.

Lin, S.-Y., Lin, J.-F., 2013. Numerical investigation of lubrication force on a spherical particle moving to a plane wall at finite reynolds numbers. International Journal of Multiphase Flow 53, 40 - 53.

Mazzei, L., Lettieri, P., 2008. CFD simulations of expanding/contracting homogeneous fluidized beds and their transition to bubbling. Chemical Engineering Science 63 (24), 5831 - 5847.

McMillan, J., Shaffer, F., Gopalan, B., Chew, J. W., Hrenya, C., Hays, R., Karri, S. R., Cocco, R., 2013. Particle cluster dynamics during fluidization. Chemical Engineering Science 100 (0), 39 - 51, 11th International Conference on Gas-Liquid and Gas-Liquid-Solid Reactor Engineering.

Mehrabadi, M., Tenneti, S., Garg, R., Subramaniam, S., 5 2015. Pseudoturbulent gas-phase velocity fluctuations in homogeneous gas-solid flow: 
fixed particle assemblies and freely evolving suspensions. Journal of Fluid Mechanics 770, 210-246.

Mohd-Yusof, J., 1996. Interaction of massive particles with turbulence. Ph.D. thesis, Cornell University.

Monchaux, R., Bourgoin, M., Cartellier, A., 2010. Preferential concentration of heavy particles: A voronoi analysis. Physics of Fluids 22 (10).

Murphy, E., Subramaniam, S., 2015. Freely cooling granular gases with shortranged attractive potentials. Phys. Fluids 27 (4), 043301.

Murphy, E., Subramaniam, S., 2016a. Binary collision outcomes for inelastic soft-sphere models with cohesion. In preparation.

Murphy, E., Subramaniam, S., 2016b. Finding measures of particle clustering for model building. In preparation.

Peskin, C. S., 2002. The immersed boundary method. Acta Numerica 11, $479-517$.

Plimpton, S., 1995. Fast parallel algorithms for short-range molecular dynamics. Journal of Computational Physics 117, 1-19.

Prosperetti, A., Oguz, H. N., 2001. Physalis: A new $o(\mathrm{~N})$ method for the numerical simulation of disperse systems: Potential flow of spheres. Journal of Computational Physics 167 (1), 196-216.

Rong, L., Dong, K., Yu, A., 2013. Lattice-boltzmann simulation of fluid flow through packed beds of uniform spheres: Effect of porosity. Chemical Engineering Science 99, 44-58. 
Royer, J. R., Evans, D. J., Oyarte, L., Guo, Q., Kapit, E., Mobius, M. E., Waitukaitis, S. R., Jaeger, H. M., 2009. High-speed tracking of rupture and clustering in freely falling granular streams. Nature 459, 1110-1113.

Shaffer, F., Gopalan, B., Breault, R. W., Cocco, R., Karri, S. R., Hays, R., Knowlton, T., 2013. High speed imaging of particle flow fields in CFB risers. Powder Technology 242, 86 - 99, selected Papers from the 2010 NETL Multiphase Flow Workshop.

Shah, M. T., Utikar, R., Tade, M., Evans, G., Pareek, V., 2013. Effect of a cluster on gas-solid drag from lattice boltzmann simulations. Chemical Engineering Science 102, 365 - 372.

Subramaniam, S., 2000. Statistical representation of a spray as a point process. Phys. Fluids 12 (10), 2413-2431.

Subramaniam, S., 2014. Stability limits for gas-solid suspensions with finite fluid inertia using particle-resolved direct numerical simulations. NSF annual report: 1134500.

Tenneti, S., Garg, R., Hrenya, C., Fox, R., Subramaniam, S., 2010. Direct numerical simulation of gas-solid suspensions at moderate Reynolds number: Quantifying the coupling between hydrodynamic forces and particle velocity fluctuations. Powder Technology 203 (1), 57-69.

Tenneti, S., Garg, R., Subramaniam, S., 2011. Drag law for monodisperse gas-solid systems using particle-resolved direct numerical simulation of flow past fixed assemblies of spheres. Intl. J. Multiphase Flow 37 (9), 10721092. 
Tenneti, S., Mehrabadi, M., Subramaniam, S., 2 2016. Stochastic lagrangian model for hydrodynamic acceleration of inertial particles in gas-solid suspensions. Journal of Fluid Mechanics 788, 695-729.

Tenneti, S., Subramaniam, S., 2014. Particle-resolved direct numerical simulation for gas-solid flow model development. Annual Review of Fluid Mechanics 46 (1), 199-230.

Tenneti, S., Sun, B., Garg, R., Subramaniam, S., 2013. Role of fluid heating in dense gas-solid flow as revealed by particle-resolved direct numerical simulation. International Journal of Heat and Mass Transfer 58 (1-2), 471479.

Tran-Cong, S., Gay, M., Michaelides, E., 2004. Drag coefficients of irregularly shaped particles. Powder Technology 139 (1), 21 - 32.

van der Hoef, M., Beetstra, R., Kuipers, J., 4 2005. Lattice-Boltzmann simulations of low-Reynolds-number flow past mono- and bidisperse arrays of spheres: results for the permeability and drag force. Journal of Fluid Mechanics 528, 233-254.

van Wachem, B., Sasic, S., 2008. Derivation, simulation and validation of a cohesive particle flow cfd model. AIChE Journal 54 (1), 9-19.

Wang, W., Li, J., 2007. Simulation of gassolid two-phase flow by a multiscale CFD approachof the EMMS model to the sub-grid level. Chemical Engineering Science 62 (1-2), 208 - 231, fluidized Bed Applications.

Wen, C. Y., Yu, Y. H., 1966. Mechanics of fluidization. Chem. Eng. Prog. Symp. Series 62, 100-111. 
Xu, Y., Subramaniam, S., 2010. Effect of particle clusters on carrier flow turbulence: A direct numerical simulation study. Flow, Turbulence and Combustion 85, 735-761.

Yang, N., Wang, W., Ge, W., Wang, L., Li, J., 2004. Simulation of heterogeneous structure in a circulating fluidized-bed riser by combining the two-fluid model with the emms approach. Industrial \& Engineering Chemistry Research 43 (18), 5548-5561.

Zimmermann, S., Taghipour, F., 2005. CFD modeling of the hydrodynamics and reaction kinetics of fcc fluidized-bed reactors. Industrial \& Engineering Chemistry Research 44 (26), 9818-9827. 